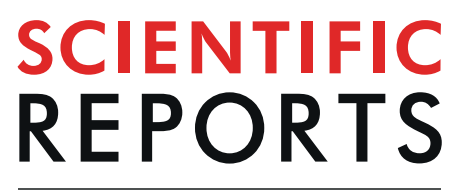

natureresearch

\title{
Occurrence, partition and environmental risk assessment of per- and polyfluoroalkyl substances in water and sediment from the Baiyangdian Lake, China
}

Rui Guo, Xiaolei Liu, Jie Liu, Yan Liu, Xiaocui Qiao, Mengyu Ma, Binghui Zheng \& Xingru Zhao*

This work examined the contamination of poly-and perfluorinated compounds (PFASs) in the water and sediment of the Baiyangdian Lake. The total concentration of PFASs in the surface water varied from 140.5 to $1828.5 \mathrm{ng} / \mathrm{L}$, and the highest concentration of PFASs were observed near the entrance of Fuhe river. The topmost contaminant was sodium perfluorohexanesulfonate ( $\mathrm{PFH} \times \mathrm{S}$ ) and perfluorooctanoic acid (PFOA) in the north and south of the Baiyangdian Lake respectively, which indicated different contamination sources. The total concentration of PFASs in the sediment varied from 0.48 to $30 \mathrm{ng} / \mathrm{g}$, and the distribution of PFASs in the sediment was similar with that in the surface water. The concentrations of polyfluoroalkyl phosphoric diesters (diPAPs) were three to four orders of magnitude lower than those of perfluorocarboxylates (PFCAs) and PFSAs. Although the pore water and the surface water had similar $\Sigma$ PFASs, the concentration of perfluorodecanoic acid (PFDA) in pore water was 1.4 to 4.4 times higher than that in surface water, and the concentration of perfluoropentanoic acid (PFPeA) in pore water was $20-70 \%$ that in surface water. The results of ecological risk assessment showed that the PFASs were currently of no immediate risk to the aquatic life.

Perfluoroalkyl and polyfluoroalkyl substances (PFASs) are anthropogenic chemicals that are both oleophobic and hydrophobic. Their excellent surface activity and repellence to water, oil, and stain made them suitable for extensive applications in fire-fighting foams, defoaming additives, adhesives, cosmetics, cleaners, coatings, paints, electronics, as well as the production of fluoropolymers. Perfluoroalkyl carboxylates (PFCAs) and perfluoroalkanesulfonates (PFSAs) have been produced and utilized for more than 50 years and are readily detected globally in water, soil, sediment, wildlife, and human blood ${ }^{1-7}$. In May 2009, the Stockholm Convention listed perfluorooctane sulfonic acid (PFOS), its salt and perfluorooctane sulfonyl fluoride as persistent organic pollutants (POPs) ${ }^{8}$.Unfortunately, short chain PFCAs and PFSAs are nowadays detected in rising concentration because they are used as replacements of PFOS and perfluorooctanoic acid (PFOA), although they are also persistent in the environment or degrade into persistent molecules ${ }^{9}$.To make things worse, short chain PFASs are harder to remove from surface water, and data are lacking on their health effect ${ }^{10}$.

In contrast, emerging per- and polyfluoroalkyl substances are much less well understood in terms of their environmental occurrence and fate. These compounds include perfluoroalkyl phosphonic acid (PFPAs), perfluoroalkyl phosphinic acids (PFPiAs), polyfluoroalkyl phosphoric acid monoesters (monoPAPs), polyfluoroalkyl phosphoric diesters (diPAPs), etc.Among them, PFPAs and PFPiAs have in fact been produced since the 1970 s and used in pesticides as wetting agents, leveling agents and defoaming agents ${ }^{11,12}$, and were among the perfluoroalkyl acids of high production volume $(4500-227000 \mathrm{~kg} / \mathrm{yr})$ in 1998 and $2002^{13}$. It was proven that PFPiAs could biodegrade into the corresponding PFPAs of the same chain length in rainbow trout ${ }^{14}$. In addition, it was suggested that PFPA and PFPiAs could be potential precursors of PFCAs. On the other hand, diPAPs have been produced since the 1970 s and used in paper, packaging materials, cosmetics, and cleaning agents ${ }^{15}$. These

National Engineering Laboratory for Lake Pollution Control and Ecological Restoration, State Environmental Protection Key Laboratory of Drinking Water Source Protection, Chinese Research Academy of Environmental Sciences, Beijing, 100012, China. *email: zhaoxr@craes.org.cn 
compounds have been detected in effluent wastewater, human serum, indoor dust, fish mussels, and raptors ${ }^{16-22}$. Unfortunately, compared to PFCAs and PFSAs, little is known about the environmental occurrence and fate of PFPAs and diPAPs, particularly in North China.

Besides insufficient data about PFPAs and diPAPs in lakes of China, Sediment-porewater partition of PFASs, which is an important process that controls the transport, fate, and ecotoxicological risk of the micro-levels of contaminants in aquatic environments are also inadequate. Studies have investigated sediment-porewater partition of polycycle aromatic hydrocarbons (PAHs) ${ }^{23}$, polychlorinated biphenyl (PCB) congeners ${ }^{24}$, Mercury and methylmercury ${ }^{25}$, etc., however, reports of PFASs in porewaters and their sediment-porewater partition were still unknown. In order to better understand the mobility and bioavailability of PFASs contained in the sediments, the concentration of these compounds in the sediment porewaters must be determined.

The Baiyangdian Lake, known as "Pearl of North China", is the largest wetland in North China. It plays an important role in flood control, water storage, climate regulation, and improvement of ecological environment. With the rapid economic development and rising population in recent years, the water quality of the Baiyangdian Lake deteriorated gradually. The Baiyangdian Lake and its surrounding areas have 39 villages with about 243,000 inhabitants, with a large amount of daily raw domestic sewage discharged into the Baiyangdian Lake. Besides, a large number of domestic sewage and industrial wastewater from the Baoding City in the upper reaches enters the Baiyangdian Lake directly via the Fuhe River. As a result of such intensive anthropogenic and industrial activities, heavy metal, organochlorine pesticides (OCPs) and polybrominated diphenyl ethers (PBDEs) have been detected in this area ${ }^{26,27}$. Though there were several publications on PFSAs levels in Baiyangdian Lake environmental samples $^{28-30}$, few reported the distribution of PFSAs between sediment and pore waters. Further more, little is known about the pollution of per- and polyfluoroalkyl substance in the past ten years, especially PFPAs, PFPiAs, and diPAPs. The current study analyzed PFASs contamination level in collected sediment, pore water, surface water samples from Baiyangdian Lake. And the partitioning of PFSAs between sediment and pore water were explored to determine the potential factors affecting their environmental behavior and fate. To our best knowledge, this is the first report of PFASs partitioning between sediment and pore water and contamination levels of PFPAs, PFPiAs and diPAPs in Baiyangdian Lake.

\section{Materials and Methods}

Chemicals and reagents. All reference and mass-labeled compounds were purchased from Wellington Laboratories (Ontario, Canada). The PFCAs standards included perfluorobutanoic acid (PFBA), perfluoropentanoic acid (PFPeA), perfluorohexanoic acid (PFHxA), perfluoroheptanoic acid (PFHpA), perfluorooctanoic acid (PFOA), perfluorononanoic acid (PFNA), perfluorodecanoic acid (PFDA), perfluoroundecanoic acid (PFUnA), perfluorododecanoic acid (PFDoA), perfluorotridecanoic acid (PFTrA), and perfluorotetradecanoic acid (PFTeA),.The PFSAs standards contained potassium perfluorobutanesulfonate (PFBS), sodium perfluorohexanesulfonate (PFHxS), sodium perfluorooctanesulfonate (PFOS), and sodium perfluorodecanesulfonate (PFDS). The PFPAs and PFPIAs standards included perfluorohexylphosphonic acid (C6-PFPA) perfluorooctylphosphonic acid (C8-PFPA), perfluorodecylphosphonic acid (C10-PFPA), sodium bis(perfluorohexyl)phosphinate (C6/ C6-PFPIA), sodium perfluorohexylperfluorooctylphosphinate (C6/C8-PFPIA), and sodium bis(perfluorooctyl) phosphinate (C8/C8-PFPIA). The DiPAPs standards contained sodium bis $(1 \mathrm{H}, 1 \mathrm{H}, 2 \mathrm{H}, 2 \mathrm{H}$-perfluorooctyl)phosphate (6:2 diPAP) and sodium bis $(1 \mathrm{H}, 1 \mathrm{H}, 2 \mathrm{H}, 2 \mathrm{H}$-perfluodecyl)phosphate (8:2diPAP). The mass-labeled internal standard (IS) included $\left[{ }^{13} \mathrm{C}_{4}\right]$-PFBA, $\left[{ }^{13} \mathrm{C}_{2}\right]$-PFHxA, $\left[{ }^{13} \mathrm{C}_{4}\right]$-PFOA, $\left[{ }^{13} \mathrm{C}_{5}\right]-\mathrm{PFNA},\left[{ }^{13} \mathrm{C}_{2}\right]-\mathrm{PFDA},\left[{ }^{13} \mathrm{C}_{2}\right]-\mathrm{PFUnDA}$, $\left[{ }^{13} \mathrm{C}_{2}\right]$-PFDoDA, $\left[{ }^{18} \mathrm{O}_{2}\right]$-PFHxS, $\left[{ }^{13} \mathrm{C}_{4}\right]$-PFOS, $\left[{ }^{13} \mathrm{C}_{4}\right]-6: 2$ diPAP, and $\left[{ }^{13} \mathrm{C}_{4}\right]-8: 2 \mathrm{diPAP}$.

LC-MS grade methanol, acetonitrile, and methyl-tert-butyl ether (MTBE) were purchased from Fisher Scientific (Fair Lawn, NJ, USA). LC grade ammonium acetate waspurchased from Fisher Scientific (Fair Lawn, NJ, USA). Analytical grade sodium hydroxide $(\mathrm{NaOH})$ was purchased from Sinopharm Chemical Reagent Beijing, Co., Ltd. Tetrabutyl ammonium hydrogen sulphate (TBAS) was purchased from J.T. Baker (Phillipsburg, NJ, USA). Oasis ${ }^{\circledR}$ weak anion exchange solid phase extraction cartridges (WAX; $6 \mathrm{cc}, 150 \mathrm{mg}$ ) were purchased from Waters (Milford, MA). Milli-Q water was used in all analytical experiments.

Sample collection. Surface water and sediment samples were collected at Baiyangdian Lake in March 2016. Surface water samples were collected with a stainless steel bucket and stored in $1 \mathrm{~L}$ polypropylene (PP) containers with a narrow mouth and a screw cap. A total of 15 surface water samples were collected. The corresponding sediment samples were collected with a bottom grab and stored in stainless steel containers.

Sample duplicates and field blanks were collected and analyzed along with laboratory and procedural blanks. The stainless steel bucket, stainless steel containers, and PP bottles were cleaned before use by rinsing sequentially with methanol, distilled water, and then water from the sampling site. All samples were kept in an ice bath during shipping, and all water samples were extracted immediately upon arrival at the laboratory. Figure 1 illustrates the sampling sites.

Sample preparation. Water samples. Both surface water samples and pore water samples were prepared. Pore water samples were collected by centrifuging the sediment samples from S3, S4, S6-S11, S13, and S15 at $10000 \mathrm{rpm}$ for $10 \mathrm{~min}$. Water samples were prepared according to previously published methods ${ }^{31}$. Water samples were filtered with Whatman GF/F glass microfiber $(0.7 \mu \mathrm{m}$ pore size, $4.7 \mathrm{~cm}$ diameter $)$ and then extracted with Oasis WAX solid phase cartridges (150 mg, 6cc, Waters, MA, USA). Before solid phase extraction, each mass-labeled PFSA (2 ng) was spiked into the water sample as internal standard. The WAX cartridge was conditioned with $1 \% \mathrm{NH}_{4} \mathrm{OH}$ in methanol $(6 \mathrm{~mL})$, followed by methanol $(6 \mathrm{~mL})$ and Milli-Q Water $(6 \mathrm{~mL})$. Water samples were passed through the conditioned cartridge at $5 \mathrm{~mL} / \mathrm{min}$, and the loaded cartridge waswashed firstly with ammonium acetate buffer $(\mathrm{pH}=4,25 \mathrm{mmol} / \mathrm{L}, 6 \mathrm{~mL})$ and then with $\mathrm{MeOH}(2 \mathrm{~mL})$. The target compounds 


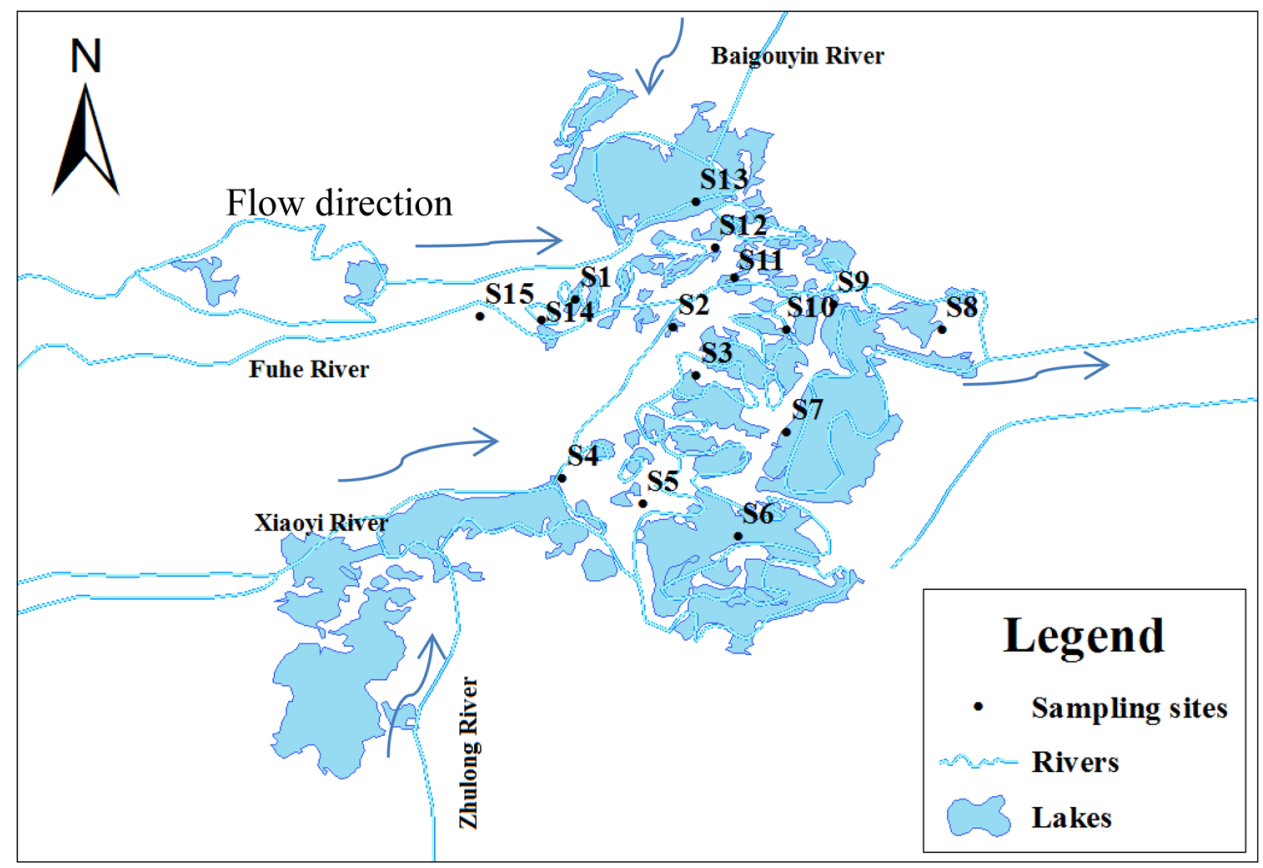

Figure 1. Sampling sites at the Baiyangdian Lake area.

were eluted firstly with $1 \% \mathrm{NH}_{4} \mathrm{OH}$ in methanol $(6 \mathrm{~mL})$ and then with $1 \% \mathrm{NH}_{4} \mathrm{OH}$ in acetonitrile $(6 \mathrm{~mL})$. The eluate was then blown to dryness with a gentle stream nitrogen gas and dispersed in methanol $(1 \mathrm{~mL})$.

Sediment samples. Sediment samples were freeze-dried, homogenized with mortar and pestle, and extracted according to the reported ion-pairing method ${ }^{32}$. Individual internal standards ( $2 \mathrm{ng}$ each) were spiked into sediment samples before extraction.

The extracts were separated into three fractions before instrumental analysis to enhance the sensitivity for target compounds. The first fraction, with which PFCAs and PFSAs were analyzed, was blended with Milli-Q water to become a solution of $1: 1 \mathrm{v} / \mathrm{v}$ methanol/water. The second fraction, with which PFPAs were analyzed, was adjusted similarly to become a 1:1 mixture of methanol and $25 \mathrm{mmol} / \mathrm{L}$ TBAS. No adjustment was made to the third fraction, with which diPAPs and PFPIAs were analyzed.

Instrumental analysis. Target compounds were separated using Ultra-Pressure Liquid Chromatography (UPLC) coupled with a Micromass Xevo-TQD mass spectrometer (UPLC-Xevo-TQD, Waters, USA) operated in the negative electrospray ionization mode. The injection volume was $10 \mu \mathrm{L}$. The analytes were separate on a Waters Acquity BEH C18 column $(50 \mathrm{~mm} \times 2.1 \mathrm{~mm}$ i.d., $1.7 \mu \mathrm{m})$ using aqueous ammonium acetate and methanol as the mobile phase with agradient elution program similar to those reported elsewhere. Multiple reactions monitoring of target compounds and optimized mass spectrum parameters were also similar to those reported elsewhere ${ }^{32}$.

Gradient elution was used to separate different compounds by liquid chromatography.For the analysis of PFCAs and PFSAs, the mobile phase consisted of (A) $10 \mathrm{mmol} / \mathrm{L}$ ammonium acetate in HPLCgrade water and (B) $10 \mathrm{mmol} / \mathrm{L}$ ammonium acetate in $8: 2(\mathrm{v} / \mathrm{v})$ methanol/acetonitrile. To analyze PFPiAs, diPAPs, and PFPAs, the mobile phase consisted of $0.1 \% \mathrm{NH}_{4} \mathrm{OH}$ in HPLC grade water $(\mathrm{A})$ and pure methanol (B). The flow rate was $300 \mu \mathrm{L} / \mathrm{min}$ and the injection volume was $10 \mu \mathrm{L}$. Multiple reaction monitoring (MRM) of target compounds and optimized mass spectrum parameters followed a reported set of conditions ${ }^{33}$.

The total organic carbon content (TOC) of water and sediment samples was analyzed using a multi N/C $2100 \mathrm{~S}$ system (AnalytikJena, Germany) with a procedure similar to those described elsewhere ${ }^{32}$.

Quality assurance and quality control. Quality assurance and control measures included field blank, travel blank, procedural blank, calibration curve, spike recoveries (both blank and matrix), and limit of quantification (LOQ). Field blanks were prepared by filling precleaned $1 \mathrm{~L}$ collection bottles with laboratory Milli-Q water that was previously determined to be free of PFSAs. Procedural blanks were analyzed with every batch of samples. Procedural and travel blanks were below the corresponding LOQs. Analyte recoveries were checked to determine the accuracy of the methods. Matrix spike recovery tests were performed for both water and sediment. To reduce instrumental background contamination from HPLC or solvents, an isolate trap column was connected between the solvent mixing cell and the six-way valve. Teflon-coated lab ware and glassware were avoided during all steps of sampling, pretreatment, and analysis to minimize contamination. The limits of quantification (LOQs) were defined as the smallest mass of injected compound that could afford a reproducible measurement of peak area within $\pm 20 \%$ of the duplicate injection. The LOQ and recoveries for each compound were shown in Table 1. The PFASs concentrations were quantified using external calibration curves consisting of a concentration 


\begin{tabular}{|c|c|c|c|}
\hline \multirow[b]{2}{*}{ Compounds } & \multirow{2}{*}{$\begin{array}{l}\text { LOQ } \\
\text { (ng/L) }\end{array}$} & \multicolumn{2}{|c|}{ Recoveries (\%) } \\
\hline & & water & sediment \\
\hline PFBA & 50 & $98.1 \pm 10.8$ & $68.8 \pm 9.6$ \\
\hline PFPeA & 50 & $81.8 \pm 8.9$ & $76.3 \pm 9.1$ \\
\hline PFHxA & 50 & $103.1 \pm 9.11$ & $75.5 \pm 10.5$ \\
\hline PFHpA & 20 & $97.2 \pm 12.0$ & $74.2 \pm 7.6$ \\
\hline PFOA & 50 & $96.8 \pm 3.3$ & $76.4 \pm 10.4$ \\
\hline PFNA & 100 & $103.5 \pm 4.6$ & $75.5 \pm 8.4$ \\
\hline PFDA & 20 & $75.6 \pm 2.9$ & $75.8 \pm 7.4$ \\
\hline PFUnA & 50 & $80.1 \pm 2.5$ & $77.3 \pm 5.4$ \\
\hline PFDoA & 20 & $107.0 \pm 7.1$ & $87.5 \pm 10.2$ \\
\hline PFTrA & 50 & $75.3 \pm 7.8$ & $102.3 \pm 3.5$ \\
\hline PFTeA & 50 & $58.6 \pm 11.5$ & $94.6 \pm 6.4$ \\
\hline PFBS & 100 & $67.5 \pm 6.8$ & $69.4 \pm 7.6$ \\
\hline PFHxS & 100 & $105.4 \pm 10.1$ & $89.6 \pm 8.5$ \\
\hline PFOS & 50 & $105.8 \pm 5.5$ & $85.4 \pm 9.8$ \\
\hline PFDS & 100 & $60.8 \pm 10.5$ & $97.6 \pm 11.5$ \\
\hline C6-PFPA & 100 & $103.9 \pm 9.1$ & $80.2 \pm 3.6$ \\
\hline C8-PFPA & 100 & $104.6 \pm 8.2$ & $75.6 \pm 2.6$ \\
\hline C10-PFPA & 100 & $89.7 \pm 10.8$ & $74.5 \pm 3.2$ \\
\hline C6/C6 PFPIA & 20 & $66.5 \pm 12.5$ & $86.6 \pm 2.5$ \\
\hline C6/C8 PFPIA & 20 & $54.2 \pm 12.2$ & $90.5 \pm 2.4$ \\
\hline C8/C8 PFPIA & 20 & $89.2 \pm 13.3$ & $85.7 \pm 2.6$ \\
\hline $6: 2$ diPAP & 20 & $108.3 \pm 8.8$ & $78.5 \pm 5.7$ \\
\hline $8: 2$ diPAP & 20 & $115.2 \pm 6.9$ & $82.4 \pm 7.6$ \\
\hline
\end{tabular}

Table 1. Limits of quantification and recoveries of target compounds.

series of $0,10,20,50,100,200,1000$, and $2000 \mathrm{ng} / \mathrm{L}$, and the deviation of every point from the regression line was less than $20 \%$ from its theoretical value.

Assessment method for environmental risk of PFASs. The environmental risk of the PFASs was assessed based on their risk quotient (RQ), currently the most commonly used measure to characterize environmental risk, calculated by dividing the measured concentration of the target substance (MEC) with the predicted no-effect concentration (PNEC):

$$
\mathrm{RQ}=\mathrm{MEC} / \mathrm{PNEC}
$$

Risk level was judged as follows: $\mathrm{RQ}<0.01$, very low risk; $0.01<\mathrm{RQ}<0.1$, low risk; $0.1<\mathrm{RQ}<1$, intermediate risk; $\mathrm{RQ}>1$, high risk.

Because toxicological data of PFASs in sediment are lacking, the sediment PNEC was calculated based on equilibrium distribution. Thus, according to the technical guidance document of the European Union for the risk assessment of chemical substances (TGD) ${ }^{34}$.

\section{Results and Discussions}

PFASs in surface water. Among the 15 analyzed PFCAs and PFSAs, ten were detected in the water samples. The concentrations of PFUnA, PFDoA, PFTrA, PFTeA and PFDS were lower than the LOQs.

Figure 2 shows the distribution of PFASs in the surface water samples from the Baiyangdian Lake. The total concentration of the PFCAs and PFSAs varied from 140.5 to $1828.5 \mathrm{ng} / \mathrm{L}$. Among the tested compounds, C6C8, C10 PFCAs and C6, C8 PFSAs were detected in all water samples. The detection rate of the PFASs with shorter chains, i.e., PFBA, PFPeA, PFNA, and PFBS,reached $66.7 \%, 80 \%, 80 \%$, and $93.3 \%$, respectively. The contaminant level of the target compounds decreased in the following order: PFHxS (range: 2.07-1688 ng/L, mean: $684 \mathrm{ng} / \mathrm{L}$ ) > PFOA (range: $13.6-441 \mathrm{ng} / \mathrm{L}$, mean: $147 \mathrm{ng} / \mathrm{L}$ ) $>$ PFBS (range: nd-51.2 ng/L, mean: $16.9 \mathrm{ng} / \mathrm{L}$ ) $>$ PFOS (range: 0.58-51.2 ng/L, mean: $15.2 \mathrm{ng} / \mathrm{L}$ ) > PFPeA (range: nd-12.7 ng/L, mean: $5.97 \mathrm{ng} / \mathrm{L}$ ) > PFHxA (range: $2.36-$ $6.12 \mathrm{ng} / \mathrm{L}$, mean: $4.84 \mathrm{ng} / \mathrm{L}$ ) > PFHpA (range: $1.16-9.5 \mathrm{ng} / \mathrm{L}$, mean: $3.16 \mathrm{ng} / \mathrm{L}$ ) > PFBA (range: $\mathrm{nd}-5.25 \mathrm{ng} / \mathrm{L}$, mean: $2.22 \mathrm{ng} / \mathrm{L}$ ) > PFNA (range: $\mathrm{nd}-1.02 \mathrm{ng} / \mathrm{L}$, mean: $0.61 \mathrm{ng} / \mathrm{L}$ ) > PFDA (range: $0.254-0.762 \mathrm{ng} / \mathrm{L}$, mean: $0.39 \mathrm{ng} / \mathrm{L}$ ). There must be different contamination sources since PFOA was dominant at S4-S7 however, PFHxS was dominant at other sites. The sites S4-S6 are near the entrance of the Xiaoyi River and the Zhulong River. As one of nine rivers entering the Baiyangdian Lake, the Zhulong River carries abundant wastewater from textile and fur plants and had a high PFOA concentration up to $8397.23 \mathrm{ng} / \mathrm{L}^{29}$. The highest concentrations of total PFASs were detected at S14, S15, and S1, which are near the entrance of the Fuhe River. The Fuhe River passes through the Baoding city and carries untreated urban sewage as it flows into the Baiyangdian Lake, and it is thus a principal source of pollution ${ }^{35}$. A photographic film production plant from one of the largest Chinese manufacturers is by the Fuhe River, and previous studies showed that the Fuhe River contained abundant PFHxS (>1000 ng/L). 


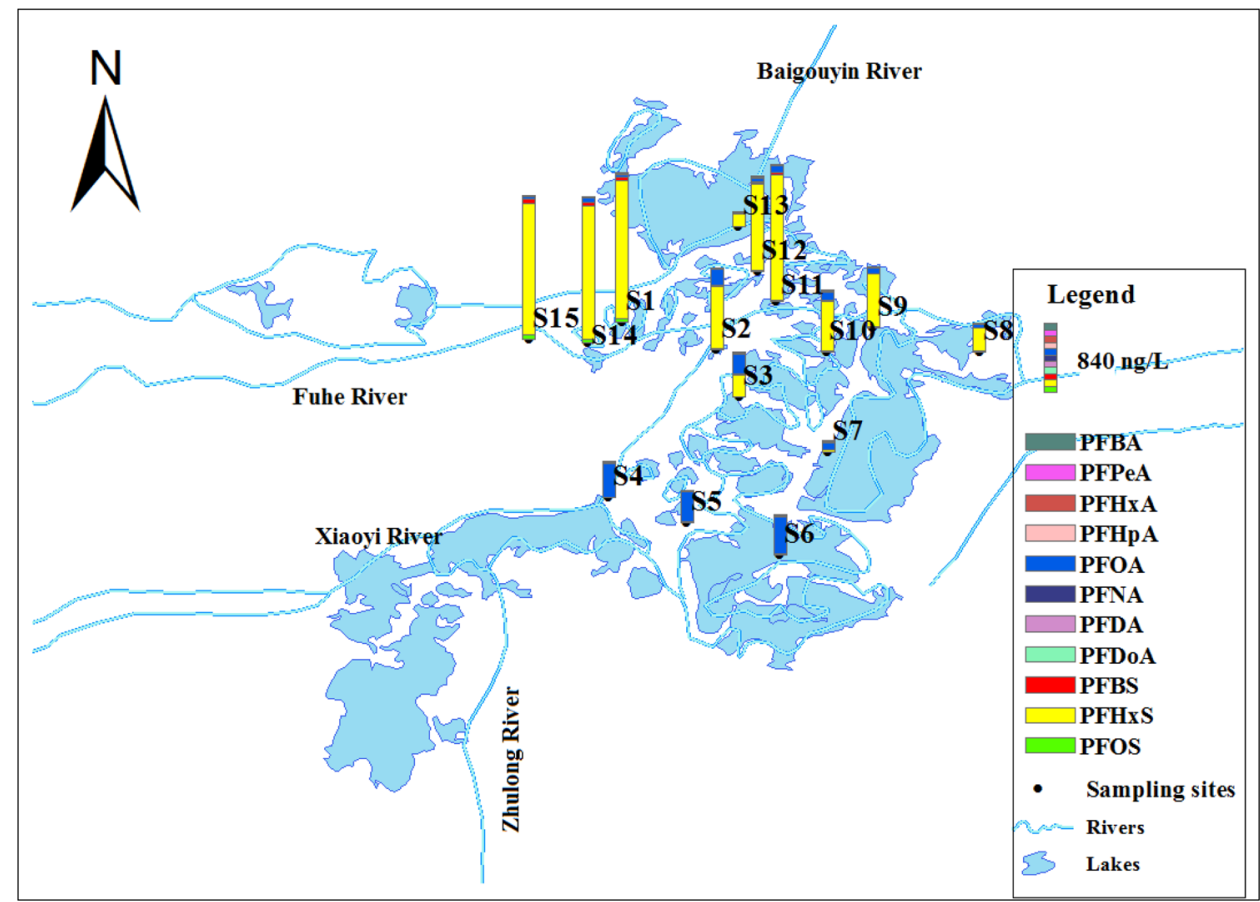

Figure 2. Distribution of PFCAs and PFSAs in Baiyangdian Lake surface water.
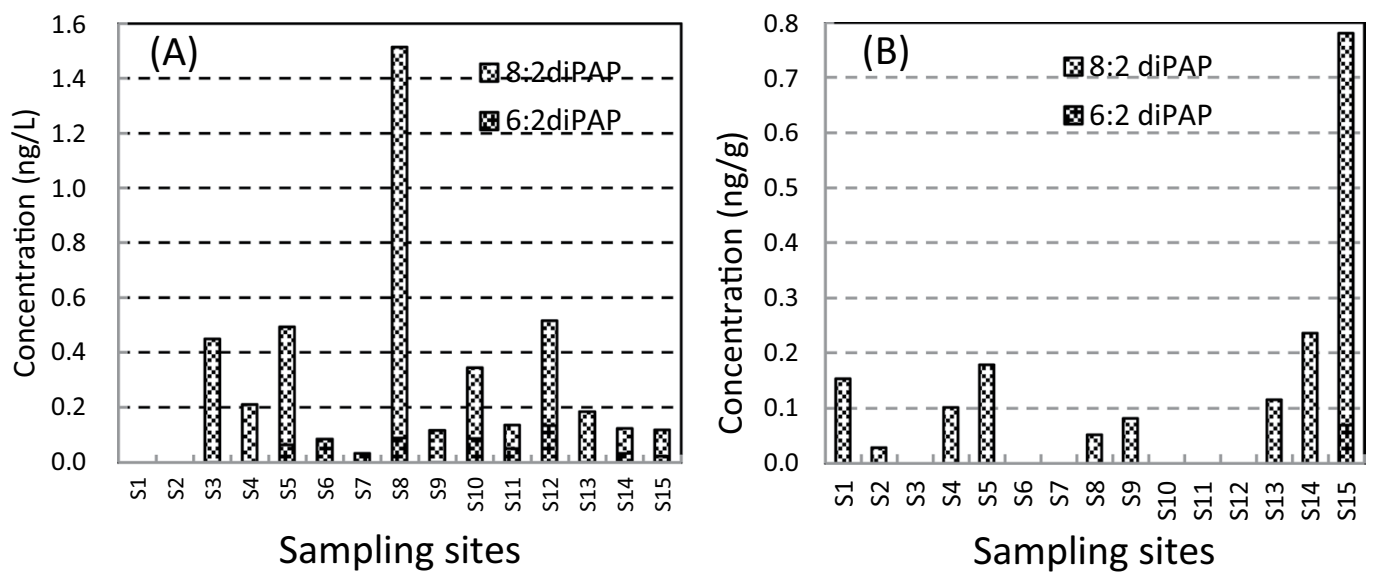

Figure 3. Distribution of diPAPs in the Baiyangdian Lake. (A) surface water, (B) sediment.

The pollutants in the samples from sites other than S4-S7 showed a similar distribution pattern (Fig. 2), which hinted on a common contamination source. The study of the Baiyangdian Lake in 2016 showed that PFOA (up to $8397 \mathrm{ng} \cdot \mathrm{L}-1$ ) and PFHxS (up to $1478 \mathrm{ng} \cdot \mathrm{L}-1$ ) were the predominant PFASs detected in the surface water ${ }^{29}$, which indicated the common contamination source of S1-S3, S8-S15. The PFSAs concentrations of the surface water collected from the Baiyangdian Lake in October 2010 ranged in 14.8-95.6 ng/L, and the lowest concentration detected in the current study was even higher than the highest concentration previously reported ${ }^{30}$. The comparison indicated that contamination of PFSAs in the Baiyangdian Lake deteriorated since 2008.

All surface water samples were free of PFPAs and PFPiAs, and 6:2 diPAP and 8:2 diPAP were detected in 60\% and $73 \%$ of the surface water samples, respectively. The concentration ranged in nd $-0.134 \mathrm{ng} / \mathrm{L}$ and $\mathrm{nd}-1.43 \mathrm{ng} / \mathrm{L}$ for 6:2 diPAP and 8:2 diPAP, respectively,three or four orders of magnitude lower than the concentrations of PFCAs and PFSAs (Fig. 3). The concentration of 8:2 diPAP was clearly much higher than that of 6:2 diPAP. The diPAPs must have come from a contamination source different from that of PFCAs and PFSAs because of their distribution characteristics and the higher concentration of 8:2 diPAP. Since PAPs are primarily used in paper products for food packaging, the diPAPs probably came from domestic sewage and household garbage ${ }^{36}$. Since the degradation of diPAPs to PFCAs can occur in wastewater treatment plants, diPAPs must be both a precursor of PFCAs and a potential fluorinated contaminant of their own ${ }^{37,38}$. To the best of our knowledge, this work is the first report that determined diPAPs in the Baiyangdian Lake. 


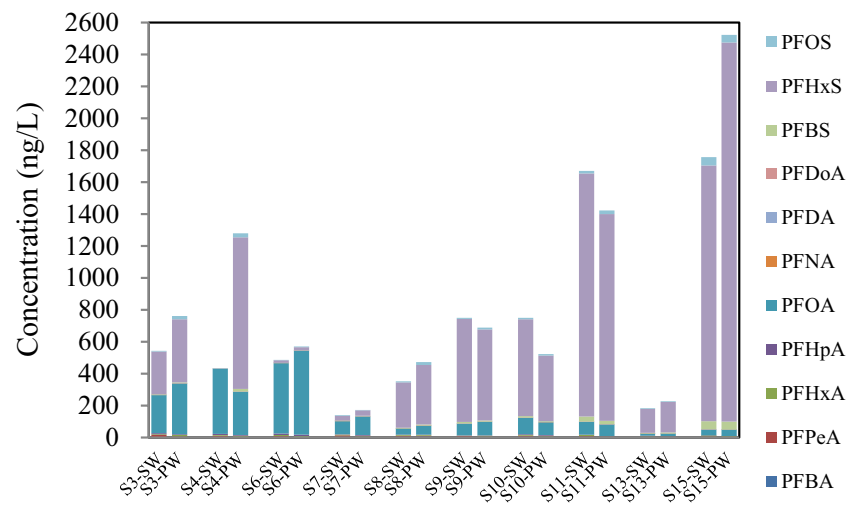

Figure 4. Comparison of total concentration of PFCAs and PFSAs in the surface water and the pore water of the Baiyangdian Lake, SW stands for surface water, PW stands for pore water.

The regression analyses of the contaminants in the surface water of the research area indicated that significant correlations $(p<0.05)$ were found between various compounds. Especially, the correlations between PFOS and PFHxS, PFOS and PFBS, as well as PFHxS and PFBS were all more than 0.93, i.e., these three compounds might share similar sources and transport routes.

PFSAs in pore water. The distribution characteristics of PFCAs and PFSAs in pore water were shown in Fig. 4. Since the content of dissolved organic matter (DOM) in pore water was usually more than one order of magnitude than that in surface water, it was excepted the total concentration of PFASs in pore water would higher than those in surface water. However, the results displayed in Fig. 4 didn't support the expectation. As shown in Fig. 4, total concentration of PFASs in pore water of S3, S4, S6, S7, S8, S13 and S15 were higher than those in correspond surface water, while in sampling sites S9, S10 and S11, the results was in the opposite.

water and the pore water of the Baiyangdian Lake, SW stands for surface water, PW stands for pore water

Figure 5 presents the distribution of each PFSA congener in the surface water and the pore water. The concentration of PFPeA was much higher in surface water than in pore water, whereas PFDA was more enriched in pore water. Interestingly, although PFBA has a shorter carbon chain and presumably higher solubility in water, it was not enriched in surface water. The distribution of PFHxA, PFHpA, PFOA, PFBS, PFHxS, and PFOS showed a varied preference between surface water and pore water. The 6:2 diPAP and 8:2 diPAP were enriched in pore water in most cases.

PFASs in sediment samples. Among the 23 target compounds, four PFCAs (PFHpA, PFOA, PFNA and PFDA), two PFSAs (PFHxS and PFOS), and both diPAPs were detected in sediment samples. Figure 6 shows the concentrations of these compounds in the sediments at 15 sampling sites of the Baiyangdian Lake. The detection rate of PFCAs and PFSAs decreased in the order of PFOS $(100 \%, 0.11-8.59 \mathrm{ng} / \mathrm{g})=$ PFOA $(100 \%, 0.16-$ $3.67 \mathrm{ng} / \mathrm{g})>$ PFHxS $(93 \%$, nd $-20.5 \mathrm{ng} / \mathrm{g})>$ PFNA $(66.7 \%$, nd $-0.11 \mathrm{ng} / \mathrm{g})>$ PFDA $(60 \%$, nd $-0.19 \mathrm{ng} / \mathrm{g})>$ PFHpA $(46.7 \%$, nd- $0.23 \mathrm{ng} / \mathrm{g})$. The distribution profile of the PFCAs and PFSAs in sediment was similar to that of water. Specifically, PFOA was dominant at S4-S7, and PFHxS was dominant in all other sediment samples. Note that PFBA, PFPeA, PFHxA, PFBuS were detected in most surface water samples with a mean concentration from 2.22 to $16.9 \mathrm{ng} / \mathrm{L}$ but not detected in sediment samples, possibly because of their low affinity to sediments.

Comparison with literature showed that the observed PFASs concentration at the Baiyangdian Lake was much higher than the $\Sigma$ PFASs of the Songhua River $(0.143-1.41 \mathrm{ng} / \mathrm{g})$, the Peal River estuary (nd-2.41 ng/g), the Nansi Lake $(0.47-1.81 \mathrm{ng} / \mathrm{g})$, and the Daliao River $(0.13-0.49 \mathrm{ng} / \mathrm{g})^{39-42}$. In whole worldwide, the $\Sigma$ PFASs in sediment varied greatly. The $\Sigma$ PFASs level in sediment of target area was similar with those in Lake Superior (nd$10.5 \mathrm{ng} / \mathrm{g}$ ), Lake Huron (nd-26.0 ng/g) and Lake Michigan (0.2-10.1 ng/g) collected in 2011 and 2012 in North America $^{43}$ and urban catchment of Singapore ( 1.6 to $\left.15 \mathrm{ng} / \mathrm{g} \mathrm{d.w}\right)^{44}$. Besides, the sediment concentrations of PFOS and PFOA were at an intermediate level compared with the situations of other parts of the world. However, the observed PFHxS concentration was at a relatively high level.

Sediment-water diffusion. The partition of organic pollutants between water and sediment affects their environmental behavior and fate, and the partition is governed by their physical and chemical properties as well as sediment characteristics such as organic carbon content, $\mathrm{pH}$, ionic strength, salinity, etc. ${ }^{45}$. In current study, we calculated the distribution coefficient $\left(K_{d}\right)$ of PFOA, PFOS and PFHxS between sediment and surface water as well as between sediment and pore water. We only calculated $K_{d}$ for PFOA, PFOS and PFHxS because their detection rate was $>90 \%$. Significant correlations $(p<0.01)$ were found between sediment TOC and the sediment-pore water $K_{d}$ of PFHxS, PFOA, and PFOS (Tables 2-4).

There was no significant correlation $(\mathrm{p}>0.01)$ between sediment TOC and the sediment-surface water $K_{d}$ of the tested compounds. Hence, after equilibrium was established for the distribution of the compoundsbetween pore water and sediment, the sediment TOC would become the dominant factor affecting the distributionof the pollutant between pore water and sediment.

The partitioning of PFASs is commonly evaluated by $K_{o c}$ as follows: 

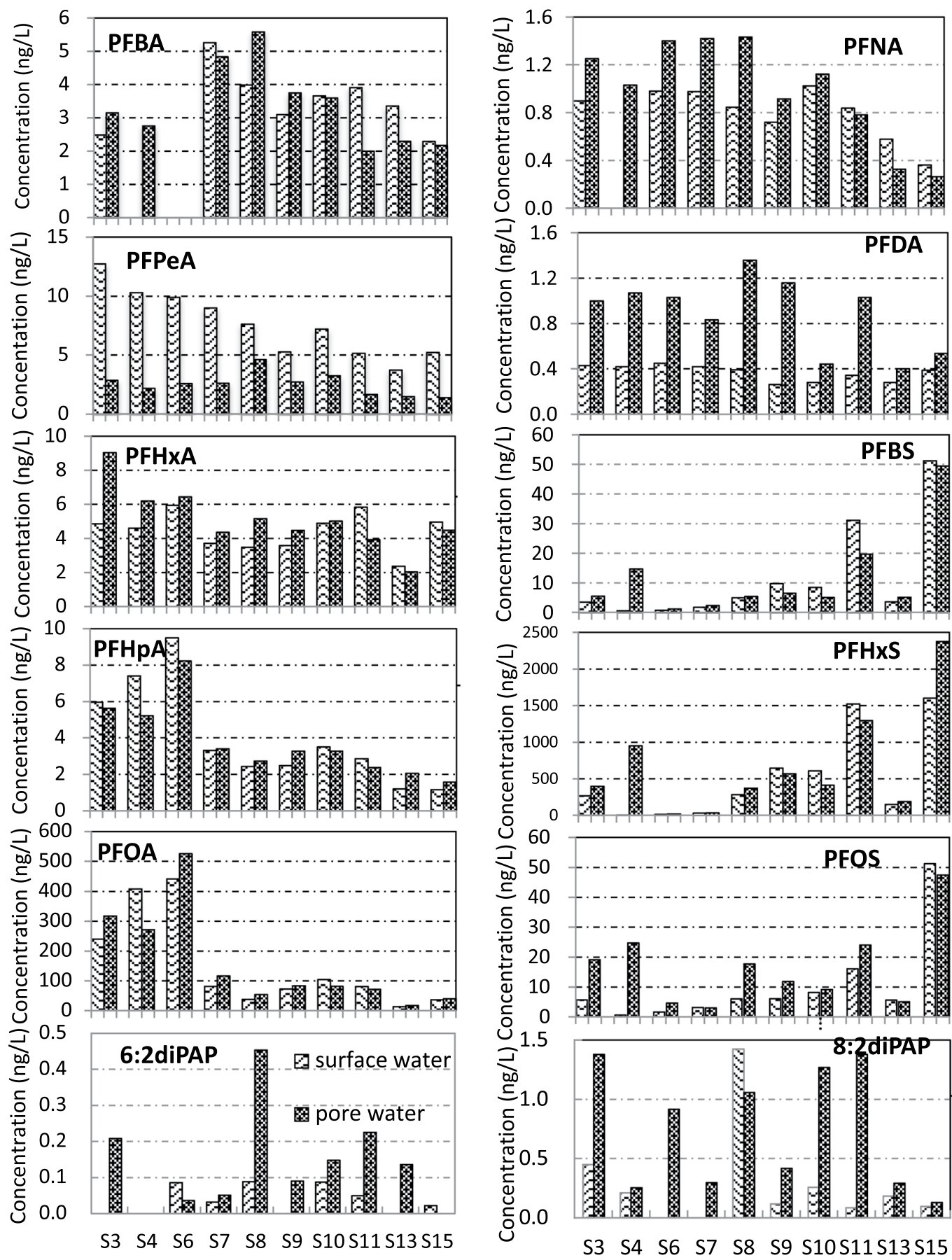

Figure 5. Distribution of PFCAs and PFSAs between surface water (sw) and pore water (pw) in the samples from the Baiyangdian Lake.

$$
\mathrm{K}_{\mathrm{oc}}=\left(\mathrm{C}_{\mathrm{s}} / C_{\mathrm{w}}\right) / f_{\mathrm{oc}}
$$

where $\mathrm{C}_{\mathrm{s}}$ is the PFASs concentration of the entire sediment based on dry weight (ng/g); $\mathrm{C}_{\mathrm{w}}$ is the PFASs concentration of surface or pore water at equilibrium $(\mathrm{ng} / \mathrm{mL})$; and $f_{o c}$ is the organic carbon fraction of the sediment (\%). The $\log K_{o c}$ values of the PFASs in pore water were: PFOA, 2.05-2.56 (mean 2.21), PFHxS, 1.82-2.32 (mean 2.09), PFOS, 2.62-3.53 (mean 3.24). The $\log K_{o c}$ values of the PFASs in surface water were: PFOA, 1.89-2.71 (mean 2.24), PFHxS, 1.94-3.28 (mean 2.38), PFOS, 3.01-4.25 (mean 3.59). It could be seen from the results of PFHxS and PFOS that each $-\mathrm{CF}_{2}$ group contributed $0.58-0.60 \log$ units to the $\log K_{o c}$ value, which was consistent with previous reports ${ }^{32,46}$. 


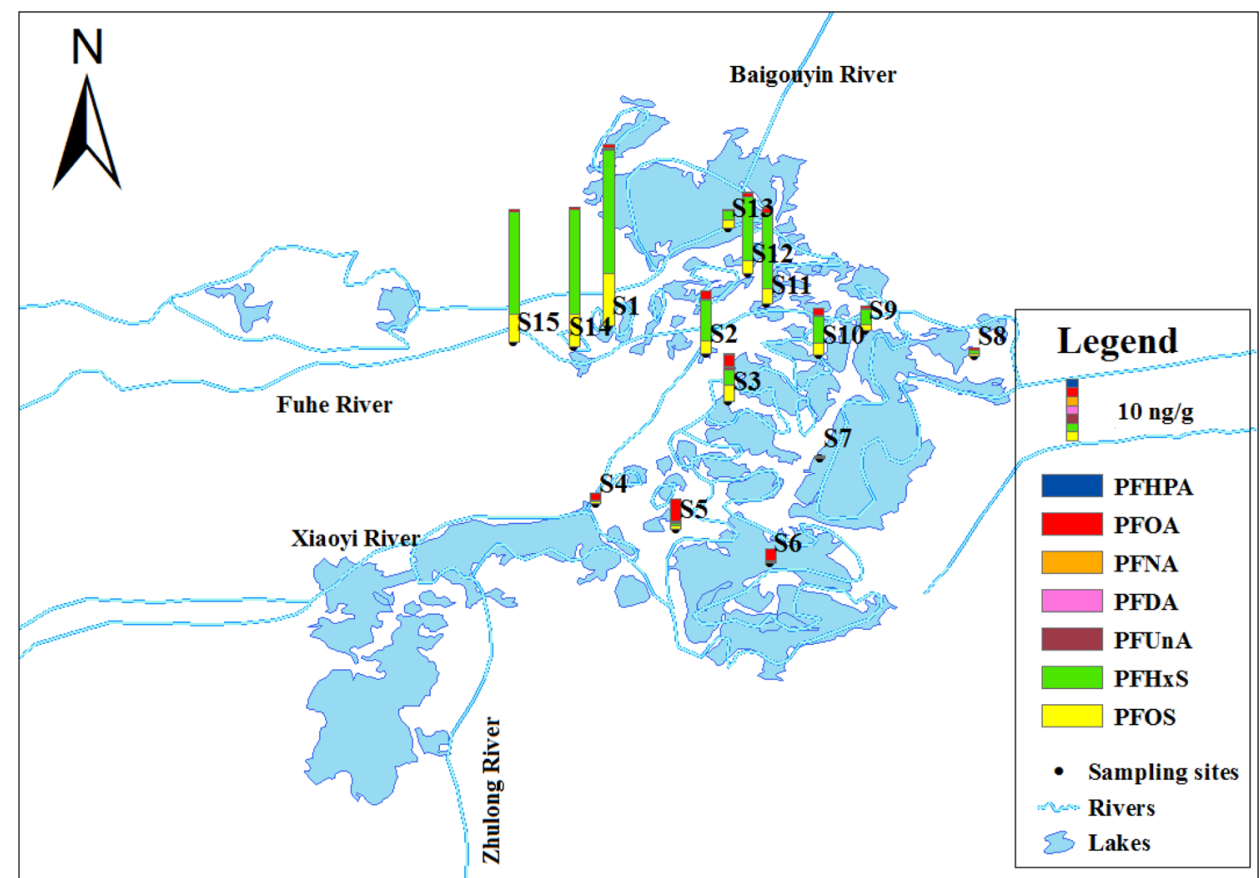

Figure 6. PFSAs contamination in sediment samples from the Baiyangdian Lake.

\begin{tabular}{|l|l|l|l|}
\hline \multicolumn{2}{|c|}{} & $\mathbf{K}_{\mathbf{d}}$ & TOC \\
\hline \multirow{4}{*}{$K_{d}$} & Pearson Correlation & 1 & $0.802(* *)$ \\
\cline { 2 - 4 } & Sig. (1-tailed) & & 0.003 \\
\cline { 2 - 4 } & $\mathrm{N}$ & 10 & 10 \\
\hline \multirow{3}{*}{ TOC } & Pearson Correlation & $0.802(* *)$ & 1 \\
\cline { 2 - 4 } & Sig. (1-tailed) & 0.003 & \\
\cline { 2 - 4 } & $\mathrm{N}$ & 10 & 10 \\
\hline
\end{tabular}

Table 2. Correlations of PFOA. **Significant correlation at $\mathrm{p}<0.01$ (1-tailed).

\begin{tabular}{|l|l|l|l|}
\hline \multicolumn{2}{|c|}{} & $\mathbf{K}_{\mathbf{d}}$ & TOC \\
\hline \multirow{4}{*}{$K_{d}$} & Pearson Correlation & 1 & $0.862(* *)$ \\
\cline { 2 - 4 } & Sig. (1-tailed) & & 0.001 \\
\cline { 2 - 4 } & $\mathrm{N}$ & 10 & 10 \\
\hline \multirow{3}{*}{ TOC } & Pearson Correlation & $0.862(* *)$ & 1 \\
\cline { 2 - 4 } & Sig. (1-tailed) & 0.001 & \\
\cline { 2 - 4 } & $\mathrm{N}$ & 10 & 10 \\
\hline
\end{tabular}

Table 3. Correlations of PFOS. **Significant correlation at $\mathrm{p}<0.01$ (1-tailed).

Environmental risk of PFASs: an assessment. The RQ of PFASs were calculated to evaluate their environmental risk.

The PNEC $_{\text {water }}$ of PFOA, PFOS, PFNA, PFHxA, and PFDA were 100, 25, 100, 97, and $11 \mu \mathrm{g} / \mathrm{L}$, respectively ${ }^{47}$.

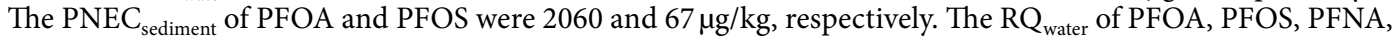
PFHxA, and PFDA were all $<0.01$, which indicated very low environmental risk. The $\mathrm{RQ}_{\text {sediment }}$ of PFOA was $<0.01$ but the $\mathrm{RQ}_{\text {sediment }}$ of PFOS ranged in $0.002-0.13$. Therefore, the environmental risk level of PFOS varied from very low to intermediate.

Since current toxicity data of PFHxS and PFBS indicate that they are less toxic than PFOS and PFOA, it was expected that they should have higher PNEC than PFOS ${ }^{48-52}$. The measured concentration of PFHxS and PFBuS in water and sediment were even lower than the PNEC of PFOS. Hence, PFHxS and PFBS in the Baiyangdian Lake did not have immediate environmental impact on the aquatic life.

The results showed that the PFASs in the Baiyangdian Lake would not generate immediate environmental impacts on the aquatic life. Nevertheless, uncertainty might arise in assessing the ecological risk of PFASs and their impact on aquatic organisms due to the scarcity of toxicity information and toxicity data of pollutants 


\begin{tabular}{|l|l|l|l|}
\hline \multicolumn{2}{|c|}{} & $\mathbf{K}_{\mathbf{d}}$ & TOC \\
\hline \multirow{4}{*}{$\mathrm{K}_{\mathrm{d}}$} & Pearson Correlation & 1 & $0.862(* *)$ \\
\cline { 2 - 4 } & Sig. (1-tailed) & & 0.001 \\
\cline { 2 - 4 } & $\mathrm{N}$ & 10 & 10 \\
\hline \multirow{3}{*}{ TOC } & Pearson Correlation & $0.862(* *)$ & 1 \\
\cline { 2 - 4 } & Sig. (1-tailed) & 0.001 & \\
\cline { 2 - 4 } & $\mathrm{N}$ & 10 & 10 \\
\hline
\end{tabular}

Table 4. Correlations of PFHxS. **Significant correlation at $\mathrm{p}<0.01$ (1-tailed).

mixture, and also because PNEC values derived from different methods may vary. As a result, comprehensive toxicological information, systematic environmental exposure data, along with realistic risk assessment method are all needed to address the adverse effects of PFASs on aquatic systems.

\section{Conclusions}

This study investigated the contamination of poly- and perfluorinated compounds (PFASs) in the water and sediment of the Baiyandian Lake with a careful analysis of their distribution, partitioning, as well as environmental hazard. The results showed that the concentration of perfluoropentanoic acid (PFPeA) was much higher in surface water than in pore water, and no significant difference was otherwise found in the total concentration of PFASs between surface water and pore water. In surface water, the total concentration of perfluoroalkyl carboxylates (PFCAs) and perfluoroalkanesulfonates (PFSAs) varied from 140.5 to $1828.5 \mathrm{ng} / \mathrm{L}$. The dominant congener was sodium perfluorohexanesulfonate (PFHxS) and perfluorooctanoic acid (PFOA) in the north and south of the Baiyangdian Lake, respectively. The highest PFSAs concentration was detected near the Fuhe River, which appeared to be an important source of contamination. All sediment samples contained perfluorooctane sulfonic acid(PFOS) and perfluorooctanoic acid (PFOA). It was found that the PFASs did not pose immediate environmental risk at the Baiyangdian Lake on the aquatic life as of the moment.

Received: 11 September 2019; Accepted: 19 February 2020;

Published online: 13 March 2020

\section{References}

1. Ahrens, L. \& Bundschuh, M. Fate and effects of poly- and perfluoroalkyl substances in the aquatic environment: A review. Environ. Toxicol. Chem. 33(9), 1921-1929 (2014).

2. Giesy, J. P. \& Kannan, K. Perfluorochemical surfactants in the environment. Environ. Sci. Technol. 36(7), 146A-152A (2002).

3. Houde, M., De Silva, A. O., Muir, D. C. \& Letcher, R. J. Monitoring of perfluorinated compounds in aquatic biota: An updated review. Environ. Sci. Technol. 45(19), 7962-7973 (2011).

4. Kato, K., Wong, L. Y., Jia, L. T., Kuklenyik, Z. \& Calafat, A. M. Trends in exposure to polyfluoroalkyl chemicals in the U.S. population: 1999-2008. Environ. Sci. Technol. 45(19), 8037-8045 (2011).

5. Lau, C. et al. Perfluoroalkyl acids: A review of monitoring and toxicological findings. Toxicol. Sci. 99(2), 366-394 (2007).

6. Rankin, K., Mabury, S. A., Jenkins, T. M. \& Washington, J. W. A North American and global survey of perfluoroalkyl substances in surface soils: Distribution patterns and mode of occurrence. Chemosphere 161, 333-341 (2016).

7. Zareitalabad, P., Siemens, J., Hamer, M. \& Amelung, W. Perfluorooctanoic acid on concentrations and distribution coefficients. Chemosphere 91(6), 725-732 (2013).

8. Stockholm Convention websites: http://chm.pops.int/Programmes/NewPOPs/Publications/tabid/695/language/en-US/Default. aspx.

9. Wang, Z., Cousins, I. T., Scheringer, M. \& Hungerbühler, K. Fluorinated alternatives to long-chain perfluoroalkyl carboxylic acids (PFCAs), perfluoroalkane sulfonic acids (PFSAs) and their potential precursors. Environ. Int. 60, 242-248 (2013).

10. Sun, M. et al. Legacy and emerging perfluoroalkyl substances are important drinking water contaminants in the Cape Fear River Watershed of North Carolina. Environ. Sci. Technol. Lett. 3(12), 415-419 (2016).

11. Heid, C., Hoffmann, D. \& Polster, J. Use of Perfluoroalkylphosphorus compounds as foam-dampening agents. United States Patent 3912654 (1975)

12. Wang, Z., Cousins, I. T., Berger, U., Hungerbühler, K. \& Scheringer, M. Comparative assessment of the environmental hazards of and exposure to perfluoroalkylphosphonic and phosphinicacids (PFPAs and PFPiAs): Current knowledge, gaps, challenges and research needs. Environ. Int. 89-90, 235-247 (2016).

13. Howard, P. H. \& Meylan, W. EPA Great Lakes study for identification of PBTs to develop analytical methods: selection of additional PBTs, use, and potential environmental release of priority chemicals - Progress Report - February 2009. In: EPA Contract No. EPW-04-019, SRC FA488, Great Lakes National Program Office. New York, U S (2009).

14. Lee, H., De Silva, A. O. \& Mabury, S. A. Dietary bioaccumulation of perfluorophosphonates and perfluorophosphinates in juvenile rainbow trout: evidence of metabolism of perfluorophosphinates. Environ. Sci. Technol. 46(6), 3489-3497 (2012).

15. US EPA: Washington, D. C. Inert Ingredient; Revocation of the tolerance exemption for mono- and bis- $(1 \mathrm{H}$, $1 \mathrm{H}$, $2 \mathrm{H}, 2 \mathrm{H}$-Perfluoroalkyl) phosphates where the alkyl group is even numbered and in the C6-C12 range; OPP-2006-0253 (2006).

16. D'eon, J. C. et al. Perfluorinatedphosphonic acids in canadian surface waters and wastewater treatment plant effluent: discovery of a new class of perfluorinated acids. Environ. Toxicol.Chem. 28(10), 2101-2107 (2009a).

17. Eriksson, U. \& Karrman, A. World-wide indoor exposure to polyfluoroalkyl phosphate esters (PAPs) and other PFASs in household dust. Environ. Sci. Technol. 49(24), 14503-14511 (2015).

18. Guo, R. et al. Determination of polyfluoroalkyl phosphoric acid diesters, perfluoroalkylphosphonic acids, perfluoroalkylphosphinic acids, perfluoroalkyl carboxylic acids, and perfluoroalkane sulfonic acids in lake trout from the Great Lakes region. Anal. Bioanal. Chem. 404(9), 2699-2709 (2012).

19. Lee, H. \& Mabury, S. A. A pilot survey of legacy and current commercial fluorinated chemicals in human sera from United States donors in 2009. Environ. Sci. Technol. 45(19), 8067-8074 (2011).

20. Yeung, L. W. Y. \& Mabury, S. A. Are humans exposed to increasing amounts of unidentified organofluorine? Environ. Chem. 13(1), $102-110$ (2016). 
21. Zabaleta, I. et al. Simultaneous determination of perfluorinated compounds and their potential precursors in mussel tissue and fish muscle tissue and liver samples by liquid chromatography-electrospray-tandem mass spectrometry. J. Chromatogr. A 1387, 13-23 (2015).

22. De Silva, A. O., Allard, C. N., Spencer, C., Webster, G. M. \& Shoeib, M. Phosphorous containing fluorinated organics:polyfluoroalkyl phosphoric diesters (diPAPs), perfluorophosphonates (PFPAs), and perfluorophosphinates (PFPiAs) in residential indoor dust. Environ. Sci. Technol. 46(22), 12575-12582 (2012).

23. Yu, Y., Xu, J., Wang, P., Sun, H. \& Dai, S. Sediment-porewater partition of polycyclicaromatic hydrocarbons (PAHs) from Lanzhou reach of Yellow River, China. J. Hazard. Mater. 165, 495-500 (2009).

24. Hawthorne, S. B., Grabanski, C. B., Miller, D. J. \& Arp, H. P. H. Improving predictability of sediment-porewater partitioning models using trends observed with PCB-contaminated field sediment. Environ. Sci. Technol. 45(17), 7365-7371 (2011).

25. Heyes, A., Miller, C. \& Mason, R. P. Mercury and methylmercury in Hudson River sediment: impact of tidal resuspension on partitioning and methylation. Mar. Chem. 90, 75-89 (2004).

26. Chen, C. Y., Pickhardt, P. C., Xu, M. Q. \& Folt, C. L. Mercury and Arsenic bioaccumulation and eutrophication in Baiyangdian Lake, China. Water, Air, Soil Pollut. 190, 115-127 (2008).

27. Hu, G. et al. Concentrations and accumulation features of organochlorine pesticides in the Baiyangdian Lake freshwater food web of North China. Arch. Environ.Contam.Toxicol. 58, 700-710 (2010).

28. Shi, Y., Pan, Y., Wang, J. \& Cai, Y. Distribution of perfluorinated compounds in water, sediment, biota and floating plants in Baiyangdian Lake, China. J. Environ. Monit. 14(2), 636-642 (2012).

29. Cui, Q. Q., Pan, Y. T., Zhang, H. X., Sheng, N. \& Dai, J. Y. Elevated concentrations of perfluorohexane sulfonate and other per- and polyfluoroalkyl substances in Baiyangdian Lake (China): Source characterization and exposure assessment. Environ. Pollut. 24, 684-691 (2018).

30. Zhou, Z., Shi, Y. L., Li, W. H., Xu, L. \& Cai, Y. Q. Perfluorinated compounds in surface water and organisms from Baiyangdian Lake in north China: Source profiles, bioaccumulation and potential risk. Bull Environ. Contam. Toxicol. 89, 519-524 (2012).

31. Liu, X. L., Liu, J., Guo, R., Zhao, X. R. \& Shen, J. S. Ultra performance liquid chromatography-tandem mass spectrometry for determination of per- and polyfluorinated compounds in surface water. Chin. J. Anal. Chem. 46(9), 1400-1407 (2018) (in Chinese).

32. Zheng, B. H. et al. Distribution characteristics of poly-and perfluoroalkyl substances in the Yangtze river delta. J. Environ. Sci. 11, 100-112 (2017).

33. Guo, R. et al. Application of a comprehensive extraction technique for the determination of poly- and perfluoroalkyl substance (PFASs) in Great Lakes region sediment. Chemosphere 164, 535-546 (2016).

34. Leeuwen, K. V. Technical guidance document on risk assessment in support of commission directive 93/67/EEC on risk assessment for new notified substances, Commission Regulation (EC) No 1488/94 on Risk Assessment for existing substances, and Directive 98/8/EC of the European Parliament and of the Council concerning the placing of biocidal products on the market. Part I-IV, European Chemicals Bureau (ECB), JRC-Ispra (VA), Italy, April. Part II European Commission Joint Research Centre EUR, 20418 (2003).

35. Yan, C. F. \& Wu, X. L. The sources of pollution control in Baiyangdian based on the ecological pollution of Fuhe river. Water Sci. Eng. Technol. 77-79 (2008) (in Chinese).

36. Gebbink, W. A., Ullah, S., Sandblom, O. \& Berger, U. Polyfluoroalkyl phosphate esters and perfluoroalkyl carboxylic acid in target food samples and packaging-method development and screening. Environ. Sci. Pollut. Res. 20(11), 7949-7958 (2013).

37. Lee, H., D'eon, J. C. \& Mabury, S. A. Biodegradation of polyfluoroalkylphosphates as a source of perfluorinated acids to the environment. Environ. Sci. Technol. 44(9), 3305-3310 (2010).

38. D'eon, J. C. \& Mabury, S. A. Production of perfluorinated carboxylic acids (PFCAs) from the biotransformation of polyfluoroalkyl phosphate surfactants (PAPs): exploring routes of human contamination. Environ. Sci. Technol. 41(13), 4799-4805 (2007).

39. Cao, Y., Cao, X., Wang, H., Wan, Y. \& Wang, S. Assessment on the distribution and partitioning of perfluorinated compounds in the water and sediment of Nansi Lake, China. Environ. Monit. Assess. 187(10), 611-620 (2015).

40. Dong, W. et al. Occurrence and partition of perfluorinated compounds (PFCs) in Water and Sediment from the Songhua River, China. Arch. Environ. Contam. Toxicol. 74(3), 492-501 (2017) (in Chinese).

41. Gao, Y. et al. Occurrence and fate of perfluoroalkyl substances in marine sediments from the Chinese Bohai Sea, Yellow Sea, and East China Sea. Environ. Pollut. 194, 60-68 (2014).

42. Gong, X. X., Li, B., Liu, Y. Y., Liu, R. X. \& Song, Y. H. Pollution levels and ecological risk assessment of typical perfluorinated compounds in riverine water and sediments of Hun River and Daliao River watershed. Acta. Sci. Circumst. 35, 2177-2184 (2015) (in Chinese).

43. Kannan, K., Tao, L. \& Sinclair, E. Perfluorinated compounds in aquatic organisms at various trophic levels in a Great Lakes food Chain. Arch. Environ. Contam. Toxicol. 48(4), 559-566 (2005).

44. Chen, H. et al. Characterization of occurrence, sources and sinks of perfluoroalkyl and polyfluoroalkyl substances (PFASs) in a tropical urban catchment. Environ. Pollut. 227, 397-405 (2017).

45. Higgins, C. P. \& Luthy, R. G. Sorption of perfluorinated surfactants on sediments. Environ. Sci. Technol. 40(23), 7251-7256 (2006).

46. Shu, W. Research on inflow and outflow river pollution chatacteristicstin lake Taihu basin. Master Degree Thesis. Chinese Research Academy of environmental Science, Beijing, China (2011) (in Chinese).

47. Li, J. et al. Risk assessment of perfluoroalkyl compounds (PFCs) in water and sediment samples of Hanjiang River. Acta. Sci. Nat. Univ. Pekin. 53, 913-920 (2017) (in Chinese).

48. Butenhoff, J. L., Chang, S.-C., Ehresman, D. J. \& York, R. G. Evaluation of potential reproductive and developmental toxicity of potassium perfluorohexanesulfonate in Sprague Dawley rats. Reprod. Toxicol. 27(3-4), 331-341 (2009).

49. Cristina, G. C. et al. In ovo effects of perfluorohexane sulfonate and perfluorohexanoate on pipping success, development, mRNA expression, and thyroid hormone Levels in Chicken Embryos. Toxicol. Sci. 127(1), 216-224 (2012).

50. Kim, S. J., Shin, H., Lee, Y. B. \& Cho, H. Y. Sex-sepcific risk assessment of PFHxS using a physiologically based pharmacokinetic model. Arch. Toxicol. 92(3), 1113-1131 (2018).

51. Sundström, M. et al. Comparative pharmacokinetics of perfluorohexanesulfonate (PFHxS) in rats, mice, and monkeys. Reprod. Toxicol. 33(4), 441-451 (2012).

52. Wei, R. G., Su, H. Q. \& Qin, Z. F. Developmental toxicity of perfluorooctane sulfonate, perfluorohexane sulfonate and perfluorobutane sulfonate to xenopus embryos. Asian J. Ecotox. 7, 542-548 (2012) (in Chinese).

\section{Acknowledgements}

This work was supported financially by the National Natural Science Foundation of China (No. 21607145) and the Special Program for the Basic Work of Science and Technology (No. 2015FY110900-6).

\section{Author contributions}

Rui Guo wrote the main manuscript text. Xiaolei Liu, Jie Liu did most of experiments. Yan Liu prepared Fig. 1 and XiaocuiQiao prepared Fig. 4. Mengyu Ma did small part of experiments. Binghui Zheng and Xingru Zhao gave constructive advice. 


\section{Competing interests}

The authors declare no competing interests.

\section{Additional information}

Correspondence and requests for materials should be addressed to X.Z.

Reprints and permissions information is available at www.nature.com/reprints.

Publisher's note Springer Nature remains neutral with regard to jurisdictional claims in published maps and institutional affiliations.

(c) (i) Open Access This article is licensed under a Creative Commons Attribution 4.0 International License, which permits use, sharing, adaptation, distribution and reproduction in any medium or format, as long as you give appropriate credit to the original author(s) and the source, provide a link to the Creative Commons license, and indicate if changes were made. The images or other third party material in this article are included in the article's Creative Commons license, unless indicated otherwise in a credit line to the material. If material is not included in the article's Creative Commons license and your intended use is not permitted by statutory regulation or exceeds the permitted use, you will need to obtain permission directly from the copyright holder. To view a copy of this license, visit http://creativecommons.org/licenses/by/4.0/.

(c) The Author(s) 2020 